\title{
Article \\ Investigation of nonlinear optical properties of quantum dots deposited onto the sample glass using time-resolved inline digital holography
} \author{
J. ${ }^{4}$, Petrov N. V. ${ }^{3 *}$ (iD

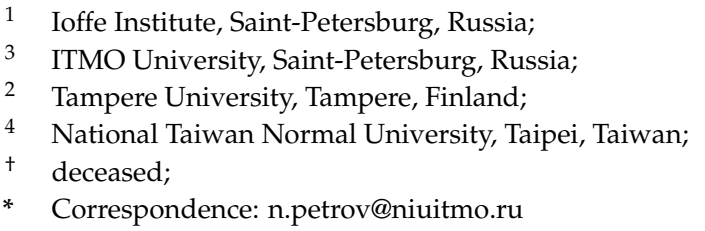

Belashov A. V., ${ }^{1}\left({ }^{\circ}\right.$, Shevkunov I. A. ${ }^{2}{ }^{\oplus}$, Kolesova E. P. ${ }^{3}$, Orlova A. O. ${ }^{3}$, Putilin S. E. ${ }^{3 \dagger}$, Veniaminov A. V. ${ }^{3}$, Cheng C.

\begin{abstract}
We report on the application of time-resolved inline digital holography to study the nonlinear optical properties of quantum dots deposited onto the sample glass. Fresnel diffraction patterns of the probe pulse due to noncollinear degenerate phase modulation induced by the femtosecond pump pulse were extracted from the set of inline digital holograms and analyzed. Absolute values of the nonlinear refractive index of both sample glass substrate and the deposited layer of quantum dots were evaluated using the proposed technique. To characterize the inhomogeneous distribution of samples' nonlinear optical properties we proposed to plot an optical nonlinearity map calculated as a local standard deviation of diffraction patterns intensity induced by noncollinear degenerate phase modulation.
\end{abstract}

Keywords: nonlinear refractive index, noncolinear degenerate phase modulation, femtosecond pulses, quantum dots, inline digital holograms, pump-probe digital holography, diffraction patterns

\section{Introduction}

Recently much attention has been paid to the investigation of the optical nonlinear properties of various samples and materials, as well as the development of high-precision techniques for implementation of such measurements. The relevance of this problem is mostly due to a large number of novel optical materials being developed in the last decade. Many studies have also reported development of novel optoelectronic devices based on the proposed materials [1-3]. One of the key material parameters highly relevant for some of these developments and responsible for the nonlinear photorefractive response is the nonlinear refractive index. In recent years, many studies and reviews were related to the estimation of this parameter in some emerging materials, including nanostructures and quantum dots (QD) [4-8]. These studies have shown that the nonlinear refractive indices of quantum dots in aggregates and nanofilms can significantly (by 3-6 orders of magnitude) exceed the nonlinear refractive indices of traditional homogeneous optical media [9-14].

Until now, several approaches to measure the nonlinear optical properties of the material have been proposed. These methods are based on Z-scan approach [5], interferometric pump-probe configuration [15], integrated digital holography for measuring thermal lens (TL) and thermal mirror (TM) effects induced by femtosecond laser pulses [16], time-resolved digital holographic microscopy $[17,18]$ etc. Meanwhile, the investigation of the spatial distribution of the samples with inhomogeneous nonlinear refractive index has been reported using point-by-point scanning of the sample $[19,20]$.

In our previous study [21] a novel time-resolved inline digital holography (TRIDH) approach was proposed for the evaluation of nonlinear refractive index of the homogeneous optical media with a constant nonlinear refractive index. The method is based on analysis of inline digital holograms set recorded at a relatively small distance from the object plane. Stepwise comparison of numerically simulated diffraction patterns obtained at various set 
of parameters with experimentally recorded data allows for determining of the samples optical properties by minimization of fitting error.

In contrast with other methods for the investigation of nonlinear optical phenomena (e.g. $[19,22-24])$, TRIDH approach can be implemented on a lensless optical setup where the pump beam is collimated [25], and no requirement of extremely high power density of a focused lased beam is needed. It provides higher stability of the optical setup and enables noninvasive investigation of the optical materials with low destruction threshold level.

In this work, we present an experimental validation of the method on a sample glass partially covered with several layers of quantum dots. The remaining part of the paper contains the following: In sections 2 and the 3, the sample preparation and experimental setup for recording of the inline digital holograms data set are described. Section 4 presents the evaluation of the glass substrate nonlinear refractive index within the area, where no QDs were deposited, similarly to the process described in [21]. Sections 5 and 6 discuss the more complex case of inhomogeneously distributed nonlinear optical properties, the evaluation of QD nonlinear refractive index, and estimation of the general "nonlinearity map" of the sample.

\section{Quantum dots sample preparation}

Colloidal CdSe/ZnS quantum dots (QD) of the core-shell type were obtained as a result of high-temperature organo-metallic synthesis by hot injection according to the protocol described in [26]. We used CdSe/ZnS QD ensembles with average core diameters within the range of $3.5-5.5 \mathrm{~nm}$. During the QD synthesis, their surface is coated with trioctylphosphine oxide (TOPO) molecules, which provide the quantum dots solubility in hydrophobic solvents and prevent spontaneous QD aggregation. The formation of nanoparticles monolayers was carried out using a KN2002 Langmuir-Blodgett Trough (KSV NIMA, Sweden). Distilled water with a conductivity of $18 \mathrm{M} \Omega / \mathrm{cm}$ was poured into a small bath. Afterward, the water surface between the barriers was cleaned using an air pump to achieve a water film surface tension of less than $0.01 \mathrm{mN} / \mathrm{cm}$ (which corresponds to a clean water surface). A Wilhelm sensor was used to measure the surface tension force. The substrates were vertically lowered into the water purified from contamination. Then the process of the water surface cleaning was repeated again. When the required purity degree was achieved, colloidal solution of nanoparticles was evenly distributed onto the water surface.

It was experimentally found that complete evaporation of hexane from the water surface takes place within 15 minutes. After distribution of the nanoparticles on the surface of the water, nanoparticles were allowed to spread evenly over the surface of the water for the next 30 minutes.Then, the film of nanoparticles was formed by reducing the film area and increasing the surface tension by moving the barriers. Using the movable surface barriers, the film can be compressed to the form of a monolayer with close packing of nanoparticles, in which the area per nanoparticle corresponds to the cross-sectional area of the nanoparticle. The transfer of the film from the water surface to the substrate was carried out by the vertical deposition technology. During the sample preparation, quantum dots were deposited only at a half of the glass substrate to provide better experimental validation of the TRIDH method.

\section{Experimental setup}

The method is based on the analysis of inline digital holograms of the probe pulse, which propagates inside the studied sample along with femtosecond pump pulse with the same wavelength but much higher energy density. The optical setup (Fig. 1 (a)) utilizes Ti: Sapphire laser system (regenerative amplifier Regulus 35F1K, Avesta Project, $2.3 \mathrm{~mJ}$ pulse energy, $35 \mathrm{fs}$ pulse duration, $800 \mathrm{~nm}$ central wavelength, $30 \mathrm{~nm}$ spectral width, 1 $\mathrm{kHz}$ repetition rate), which beam is split into the pump and probe beams, $96 \%$ and $2 \%$ of total energy, respectively. These beams are crossing in the horizontal plane under an adjustable acute angle $\beta \approx 30^{\circ}$ and overlapped inside the investigated sample $S$. Due to 
noncollinear degenerate phase modulation (NDPM) of the probe wave it diffracts at a moderate distance between the sample and image planes as demonstrated in Fig. 1 (a). If the desired defocused distance is very small (in our case $\approx 2 \mathrm{~mm}$ ), $4 \mathrm{f}$ lens system can be used for transferring the sample plane closer to the CMOS1 sensor $(1024 \times 768$ pixels, $\gamma_{m . c .} \times 2.8 \mu \mathrm{m}$, where $\gamma_{m . c} \approx 2$ is $4 \mathrm{f}$ system magnification coefficient). Another CMOS2 sensor $(1900 \times 1200,5.86 \mu \mathrm{m})$ was placed directly after the sample at the higher distance (in our case $\approx 15 \mathrm{~cm}$ ) to record the far-field diffraction pattern. Due to the small intersection area between the two femtosecond pulses in off-axis configuration (also known as walk-off effect [27]) intersection area between the probe and pump pulses is not very large and is slightly shifting during their propagation inside the sample (see semitransparent gray area in Fig. $1(b))$.
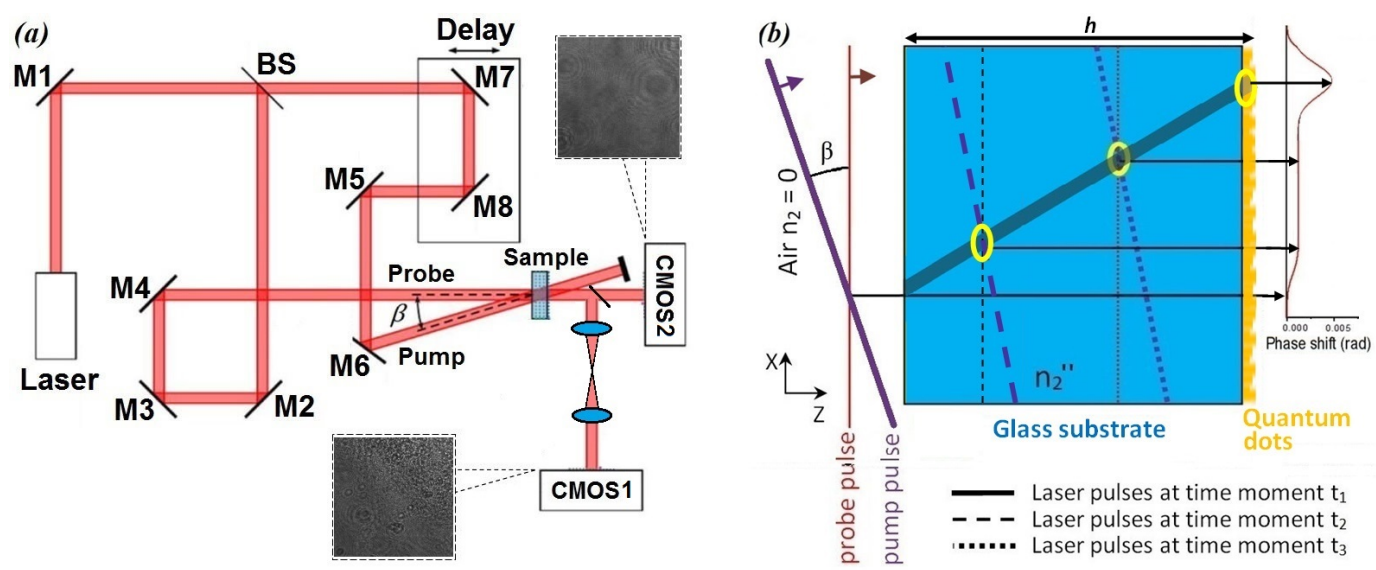

Figure 1. (a) is a scheme of the experimental setup for the recording of inline digital holograms data set in TRIDH experiment. (b) is a scheme of two femtosecond laser pulses propagation inside the sample and probe pulse NDPM-induced phase shift in XZ plane. Pulses propagation is from left to right according to red and purple arrows. Location of both femtosecond pulses in three time moments are denoted with a solid, dash, and dotted lines. The plot on the right indicates the amount of phase shift induced to probe pulse at each point of the X-axis. Gray semitransparent area indicates the pulses intersection area, blue and orange colors indicate glass substrate and deposited quantum dots.

Due to the rapid relaxation of refractive index gradient induced by the presence of the pump pulse, deformation of the probe wave and accumulation of the NDPM-induced phase shift takes place only within a local area, where the two pulses actually exist at the same time. Propagation of the probe pulse from the exit plane of the sample to the image plane results in the generation of a specific diffraction pattern, which carries information about the probe pulse phase shift induced during NDPM. Variation of the pump pulse delay line results in a shift of the two pulses intersection area and thus sample can be scanned along the $\mathrm{X}$-axis. In order to increase the image quality, each inline hologram was averaged over 20 frames. Experimental data acquisition included the recording of two sets of inline digital holograms by means of two CMOS sensors with sample scanning using the pump pulse delay line. Note that for correct data processing it is important to provide low vibrations level, high sample stability, and constant CMOS sensors exposure time as well as the constant power density of pump and probe beams.

\section{Analysis of probe pulse inline holograms due to NDPM inside the glass substrate}

As we noted above, variation of the pump pulse optical path length results in a shift of the intersection region of the probe and pump pulses inside the sample under study. Therefore shift of the pump pulse delay line leads to displacement of the probe beam diffraction pattern, induced by the phase shift introduced during noncolinear degenerate phase modulation inside the sample with nonlinear refractive index $n_{2}(x, y, z)$. The simplest case 
of such NDPM-induced diffraction can be observed when the major optical characteristics (including nonlinear refractive index) are uniform within the considered area of the sample. In this case, as we described in [21], the diffraction pattern extracted from probe beam is a set of straight vertical interference fringes. In general, such a diffraction pattern resembles the diffraction of a coherent plane wave on a slit.

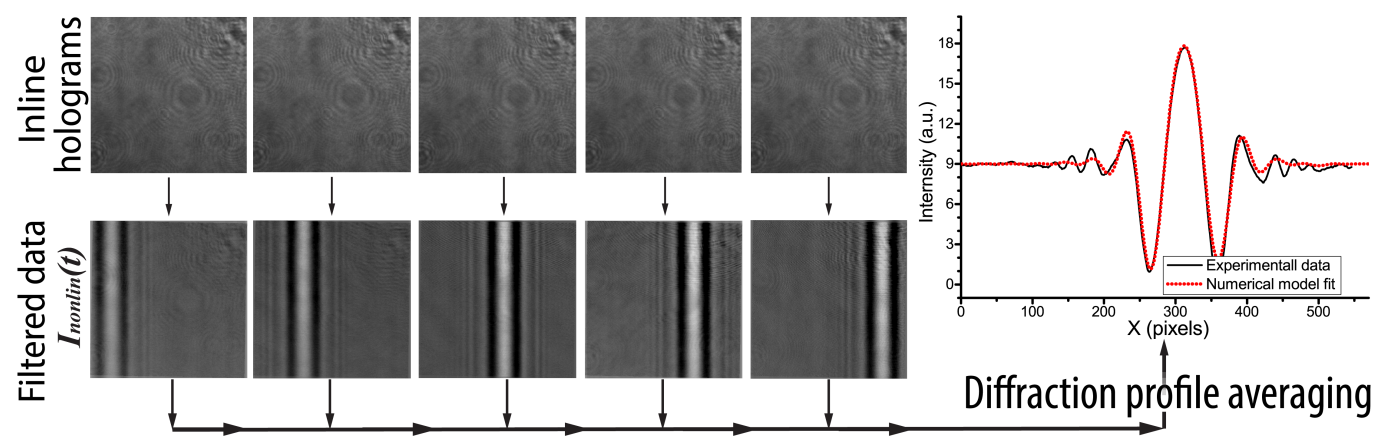

Figure 2. Examples of initial inline digital holograms before (top row) and after (bottom row) images filtration. The plot on the right side demonstrates the experimental profile and its fitting using numerically simulated data.

A similar pattern was also observed in the far-field diffraction zone when analyzing the area of the studied sample without deposited quantum dots (in this case, a sample glass with a thickness of $1 \mathrm{~mm}$ made of BK-7 glass) (Fig. 2). The figure demonstrates a set of diffraction patterns corresponding to different values of the pump pulse delay line, which results in a horizontal displacement of the diffraction pattern clearly visible in the bottom row in Fig. 2. For better visualization and data processing we subtracted the "background" intensity distribution which does not depend on the pump pulse delay line. Visualization 1 demonstrates a set of initial inline digital holograms corresponding to the movement of the pump pulse delay line.

Numerical analysis of the obtained set of time-resolved inline digital holograms described in our previous work [21] allowed us to obtain the averaged diffraction profile and fit it using the numerical model (see right inset in Fig. 2). The data processing allowed us to evaluate the absolute value of nonlinear refractive index of the sample glass as $\left|n_{2}^{\text {glass }}\right| \approx 6.3 \cdot 10^{-16} \mathrm{~cm}^{2} / \mathrm{W}$, which is in a good agreement with $n_{2}$ values, published in the literature $[21,28]$.

\section{Analysis of probe pulse diffraction due to NDPM on the quantum dots deposited onto the sample glass}

For further investigation of nonlinear optical properties of the studied sample, we utilized the set of inline digital holograms recorded using the CMOS sensor with a small diffraction distance. It allowed us to obtain the robust diffraction patterns of the probe pulse NDPM on the quantum dots (QD), deposited onto the glass substrate. In this part of the study, the glass was fixed in such a way that the upper part of the imaged sample contained a quantum dots layer whereas the lower part of the sample was a pure glass substrate, though a small amount of QD were actually deposited there by accident.

\subsection{General type of inline digital hologram observed in the experiment}

In our previous work [29], we discussed two cases of inhomogeneous distributions of the nonlinear refractive index and analysis of the obtained data for evaluation of nonlinear optical properties of the sample under study. In particular, we have considered the cases of multilayer medium, as well as a sample consisting of a homogeneous substrate and deposited rare small particles, representing local inhomogeneities of the nonlinear refractive index.

In the case of a relatively low concentration of such local inhomogeneities, when a set of inline digital holograms is recorded in the near-field zone, individual local inhomogeneities 
of nonlinear refractive index can be distinguished from each other and their diffraction patterns can be analyzed separately. In this case, it is possible to consider the diffraction profiles induced by NDPM on each individual "particle" and to evaluate nonlinear optical properties of the inhomogeneties by estimation of the diffraction pattern modulation amplitude and its asymmetry (see Sections 4B1 and 4B2 in [29]). Such a set of inline digital holograms was obtained recently when analyzing a sample glass with rare graphene particles deposited onto it. Examples of such inline digital holograms were presented in Fig. 1 (c) in [30] and will be considered in detail in our future works. In this case, numerical simulation of probe pulse NDPM on such a local nonlinear refractive index inhomogeneity [29] it is possible to find appropriate experimental parameters and to fit the experimentally recorded diffraction profile by numerically simulated diffraction curve as demonstrated in Fig. 1 (d) of our previous work [30].

However, in the case of a high concentration of nonlinear refractive index inhomogeneities, the diffraction pattern induced by NDPM of the probe pulse inside the sample is a rather chaotic intensity distribution, where no individual local inhomogeneities of nonlinear refractive index can be distinguished. In this study due to the high concentration of the quantum dots deposited onto the glass substrate (there were actually several overlapped QD layers), we observed this type of probe pulse inline holograms (see Fig. 3 (a)).

\subsection{Evaluation of absolute nonlinear refractive index of quantum dots deposited onto the glass substrate}

Due to the differences between the refractive and absorption indexes of the quantum dots and the environment, some diffraction of the probe wave was observed even in the absence of a pump pulse (Fig. 3(b)). However, when the pump pulse is present, the recorded inline digital hologram (Fig. $3\left(\right.$ a)) $I \approx I_{\text {lin }}+I_{\text {nonlin }}$ consists of two diffraction patterns: 1 is the "basic" diffraction pattern $I_{l i n}$ induced by probe wave diffraction on the inhomogeneties of linear optical properties, i.e. absorption and refractive indexes of the sample (Fig. 3(b)); 2 is the diffraction pattern of the probe wave $I_{\text {nonlin }}$ due to NDPM by the pump pulse in the area of their intersection inside the sample (Fig. $3(\mathrm{c})$ ). In this case, the diffraction caused by noncollinear degenerate phase modulation (NDPM) appears as a moving local distortion of the "basic" diffraction pattern when varying the pump pulse delay line. An example of such a set of diffraction patterns can be found in the Visualization 2.
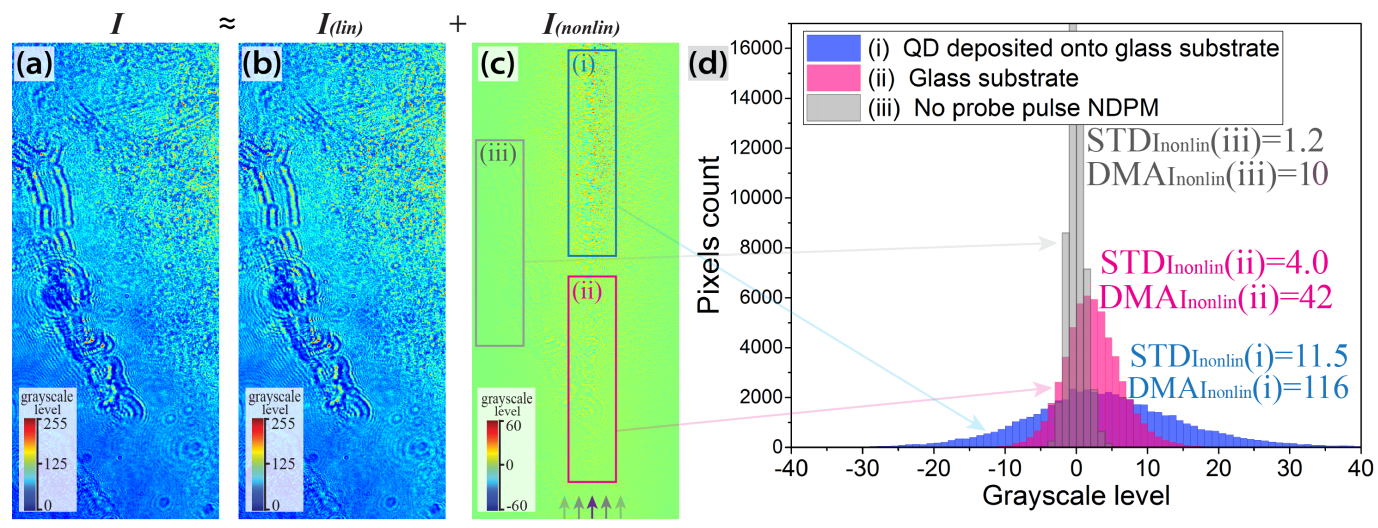

Figure 3. Examples of: (a) inline digital hologram recorded at the delay time $t_{1}$, (b) "basic" diffraction pattern $I_{\text {lin }}$ as observed without pump pulse, (c) diffraction pattern of the probe pulse $I_{\text {nonlin }}$ induced by its NDPM on the pump pulse in the area of their intersection inside the sample (purple arrows indicate the area of two pulses intersection). Notice different legends ranges in the figures. (d) represents examples of grayscale level histograms within the three areas of $I_{\text {nonlin }}$ diffraction pattern of: (i) quantum dots deposited on the glass substrate, (ii) pure sample glass only, (iii) no intersection between pump and pulses inside the sample. Standard deviations of the histograms $S T D_{I_{\text {nonlin }}}$ and diffraction pattern modulation amplitude $D M A$ are indicated for the three areas. 
In this case, due to the complex arrangement of the quantum dots on the sample glass surface, it is not possible to accurately simulate the probe pulse NDPM and diffraction pattern. However, a rough estimation of nonlinear optical properties of the sample can be performed by analysis of the diffraction pattern induced by NDPM $I_{\text {nonlin }}$ after its separation from the "basic" diffraction resulting from inhomogeneity of linear optical properties. In order to do that, the "basic" diffraction pattern $I_{l i n}$, recorded in the absence of the pump pulse should be subtracted from each of the inline digital holograms sets. The obtained set of intensity distributions can be further filtered from shot and coherent noise if required. An example of the final pattern $I_{\text {nonlin }}$ is demonstrated in Fig. 3 (c). The purple arrows indicate the area where intersection of the probe and pump pulses inside the sample took place at given delay $t_{1}$.

Figure 3 (c) clearly shows a chaotic diffraction pattern in the upper part of the sample, where quantum dots have been deposited onto the sample glass. It should be noted that the diffraction pattern in the upper part of the image is due to the probe pulse NDPM inside both: the sample glass substrate and quantum dots layer. Due to the chaotic structure of the probe pulse diffraction on a quantum dots layer, accurate analysis of such a diffraction pattern is not possible. However, its nonlinear properties can be roughly estimated by evaluation of the typical diffraction pattern modulation amplitude (DMA) within the area as a difference between the maximum and minimum intensity $D M A_{I_{\text {nonlin }}} \approx$ $\max \left(I_{\text {nonlin }}\right)-\min \left(I_{\text {nonlin }}\right)$ and its further comparison with the diffraction response of a clean substrate in the lower part of the sample. Taking into account the fact that diffraction pattern modulation amplitude is roughly proportional to the induced probe wave phase shift (see section 4B1 in [29]) absolute value of nonlinear refractive index can be estimated assuming apriori known thickness of the layer:

$$
D M A \sim \varphi_{\text {nonlin }} \approx P \frac{2 \pi}{\lambda} \int_{0}^{h}\left|n_{2}(z)\right| d z,
$$

where $P$ is the power density of the pump pulse and $h$ is the total thickness of the object. In order to do this one can evaluate $D M A_{Q D}$ value inside a local rectangular area and compare it with $D M A_{\text {ref }}$ value, detected in the 'reference' area, corresponding to the pure glass substrate with previously found nonlinear refractive index $n_{2}^{\text {glass }}$. However, both maximum and minimum intensity values within a local area ( $2 l \times 2 l$ pixels) are subject to high impact of shot noise, so to increase the robustness of the approach we suggest to analyse whole statistical distribution of the intensity values within the area and to evaluate its standard deviation, which is less susceptible to random errors:

$$
S T D_{I_{\text {nonlin }}}(x, y)=\sqrt{\frac{\sum_{i \in[x-l, x+l] \cap[y-l: y+l]}^{n} I_{\text {nonlin }}(i)-\overline{I_{\text {nonlin }}}}{n}}
$$

The examples of histograms and the result of $S T D_{I_{\text {nonlin }}}$ evaluation in three large areas of the $I_{\text {nonlin }}$ are presented in Fig. 3 (d). The three areas correspond to the regions with: (i) quantum dots deposited on the glass substrate, (ii) pure sample glass only (although a small amount of QD was deposited there by accident), (iii) no intersection between pump and probe pulses inside the sample. Note that the minor variation of 1.2 in $I_{\text {nonlin }}$ in the (iii) area is due to shot and coherent noise, although it is significantly smaller than $S T D_{I_{\text {nonlin }}}(i)=11.5$ and $S T D_{I_{\text {nonlin }}}(i i)=4.0$ in the (i) and (ii) areas. Since diffraction within the (i) area is due to both NDPM in the glass substrate and quantum dots and $S T D_{I_{\text {nonlin }}}$ (ii) corresponds to the NDPM in glass substrate only, the phase shift, induced by quantum dots layer is $\left(S T D_{I_{\text {nonlin }}}-S T D_{I_{\text {nonlin }}}\right) / S T D_{I_{\text {nonlin }}} \approx 1.88$ times higher than that of glass substrate $\left(\varphi_{n_{2}^{Q D}} / \varphi_{n_{2}^{\text {glass }}} \approx 1.88\right)$. Taking into account nonlinear refractive index of the glass substrate $\left|n_{2}^{\text {glass }}\right| \approx 6.3 \cdot 10^{-16} \mathrm{~cm}^{2} / \mathrm{W}$, its thickness $h^{\text {glass }}=1 \mathrm{~mm}$ and the thickness of quantum dots, deposited onto the sample glass $h^{\text {QDlayer }}=200 \mathrm{~nm}$, nonlinear refractive index of quantum dots layer can be evaluated as: 


$$
\left|n_{2}^{\text {QDlayer }}\right|=\frac{S T D_{I_{\text {nonlin }}}-S T D_{I_{\text {lin }}}}{S T D_{I_{\text {lin }}}} \cdot \frac{\left|n_{2}^{\text {glass }}\right| \cdot h^{\text {glass }}}{h^{\text {QDlayer }}} .
$$

The obtained value of $\left|n_{2}^{\text {QDlayer }}\right| \approx 5.9 \cdot 10^{-12} \mathrm{~cm}^{2} / \mathrm{W}$ agrees with the data about nonlinear optical properties of similar CdSe/ZnS quantum dots reported in literature [11-14] taking into account somewhat different sample preparation protocols, size of quantum dots and other experimental parameters.

\section{Evaluation of the optical "nonlinearity map"}

Besides the performed estimates of the general nonlinear refractive index of QD, one can evaluate spatial distribution representing the optical nonlinearity of the sample. In order to do so, diffraction modulation amplitudes of multiple small areas around each pixel should be analyzed (e.g. rectangular or circular area with an area of 100-500 pixels). It can be done by considering intensity histograms within the areas and the calculation of $S T D_{I_{\text {nonlin }}}$ values as performed in section 5.2. Note that larger analyzing areas result in a decrease of the spatial resolution, although a higher amount of processed pixels enhance the accuracy of the method.

When such a "map" is calculated for a single inline digital hologram, the data on only a small portion of the sample area is obtained. The area is limited by intersection region between the pump and probe pulse. In order to extend the information about the sample, the process should be repeated for other inline digital holograms with shifted intersection regions (see left part of Fig. 4). Finally, the obtained images should be combined and a single "nonlinearity map" can be constructed. In fact, such an image represents an integral amount of nonlinear refractive index of the sample $\int_{0}^{h}\left|n_{2}(z)\right| d z$ which affects the probe pulse, when it propagates across the sample.

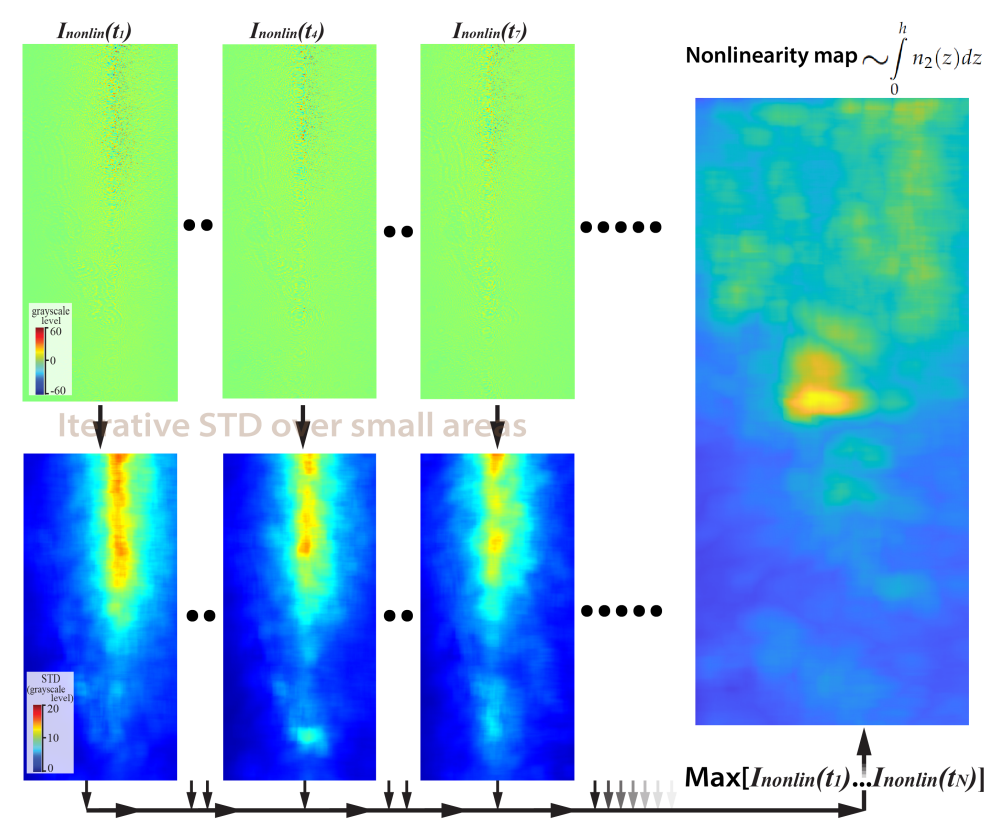

Figure 4. The top row demonstrates diffraction patterns, induced by the femtosecond probe pulse NDPM. The bottom row shows several typical nonlinearity "maps" obtained for different pump pulse delay values. The final nonlinearity "map", estimated from their combined analysis is shown in the right part of the figure.

In the case of our experimental data processing, several inline digital holograms data sets were recorded with slightly varying the defocusing distance within the range of 1-3 mm. Each of the sets was processed according to the above-mentioned protocol and afterward an averaging of the obtained nonlinearity maps was performed. The final nonlinearity 
map of the sample is presented in Fig. 4. The resulting figure clearly shows a significant difference in estimated nonlinearity within the top and bottom parts of the sample. This result well agrees with the sample preparation procedure, assuming only partial quantum dots deposition.

\section{Discussion}

In this paper, for the first time, we presented the experimental implementation of time-resolved inline digital holography for the analysis of nonlinear optical properties of a sample glass with deposited quantum dots layer. Several sets of inline digital holograms were recorded according to the TRIDH approach on the pure glass substrate area of the sample and within the region where quantum dots were deposited onto the surface. Analysis of the obtained inline digital holograms along with a priori knowledge of the sample glass thickness and quantum dots layer thickness allowed us to evaluate their nonlinear refractive indexes. The obtained results of BK-7 nonlinear refractive index absolute value $\left|n_{2}(B K 7)\right|=6.3 \cdot 10^{-16} \mathrm{~cm}^{2} / \mathrm{W}$ and absolute value nonlinear refractive index of the quantum dots $\left|n_{2}(Q D)\right|=5.9 \cdot 10^{-12} \mathrm{~cm}^{2} / \mathrm{W}$ well agrees with the typical data of these kinds of materials presented in literature. Taking into account that the quantum dots layer thickness could be nonuniform across the sample and nonlinear refractive indexes of QD may not be constant and depend e.g. on their size varying within a certain range [11-14,31], we have evaluated two-dimensional nonlinearity map within a small sample area. The final "nonlinearity map" represents the integral amount of phase shift into probe wavefront propagating across the sample. The obtained "nonlinearity map" (see Fig. 4) allowed to clearly distinguish between the areas, where quantum dots were actually deposited onto the sample glass (upper part of the image) and where no quantum dots were present (lower part of the image).

Author Contributions: Samples preparation, A.O.O. and E.P.K.; conceptualization and methodology, A.V.B. and N.V.P.; experimental investigation, I.A.S., S.E.P.; data processing, A.V.B. and I.A.S., original draft preparation, A.V.B., N.V.P. and A.O.O.; writing - review and editing, N.V.P., A.V.V., C.J.C. and A.O.O.; supervision, N.V.P. and C.J.C. All authors have read and agreed to the published version of the manuscript.

Funding: Russian Foundation for Basic Research, Project No 19-52-52018 and Ministry of Science and Technology, Taiwan (108-2923-E-003-001-MY3. A.V.V. is grateful for the support of the Ministry of Science and Higher Education of the Russian Federation, goszadanie no. 2019-1080.

Data Availability Statement: Data is contained within the article and in supplementary materials.

Conflicts of Interest: The authors declare no conflict of interest.

\section{References}

1. Mbarak, H.; Kodeary, A.; Hamidi, S.; Mohajarani, E.; Zaatar, Y. Control of nonlinear refractive index of AuNPs doped with nematic liquid crystal under external electric field. Optik 2019, 198, 163299.

2. Bairy, R.; Haleshappa, D.; Murari, M. The structural, linear and nonlinear optical properties of high-quality Zn1-xPbxO nanostructured thin films for optoelectronic device applications. Appl. Phys. B 2021, 127, 1-13.

3. Montagna, M.; Selleri, S.; Zoboli, M. Nonlinear refractive index in erbium-doped optical amplifiers. Opt. Quantum Electron. 1995, $27,871-880$.

4. Flory, F.; Escoubas, L.; Berginc, G. Optical properties of nanostructured materials: a review. J. Nanophotonics $2011,5,052502$.

5. Zhang, H.; Virally, S.; Bao, Q.; Ping, L.K.; Massar, S.; Godbout, N.; Kockaert, P. Z-scan measurement of the nonlinear refractive index of graphene. Opt. Lett. 2012, 37, 1856-1858.

6. Mai, H.H.; Kaydashev, V.E.; Tikhomirov, V.K.; Janssens, E.; Shestakov, M.V.; Meledina, M.; Turner, S.; Van Tendeloo, G.; Moshchalkov, V.V.; Lievens, P. Nonlinear Optical Properties of Ag Nanoclusters and Nanoparticles Dispersed in a Glass Host. J. Phys. Chem. C 2014, 118, 15995-16002. doi:10.1021/jp502294u.

7. Golovan, L.; Timoshenko, V.Y. Nonlinear-optical properties of porous silicon nanostructures. J. Nanoelectron. Optoelectron. 2013, 8, 223-239.

8. Zhang, Y.x.; Wang, Y.h. Nonlinear optical properties of metal nanoparticles: a review. RSC Adv. 2017, 7, 45129-45144.

9. Henari, F.Z.; Dakhel, A.A. Linear and nonlinear optical properties of gold nanoparticle-Eu oxide composite thin films. J. Appl. Phys. 2008, 104, 033110. doi:10.1063/1.2967711. 
10. Bai, S.; Li, Q.; Zhang, H.; Chen, X.; Luo, S.; Gong, H.; Yang, Y.; Zhao, D.; Qiu, M. Large third-order nonlinear refractive index coefficient based on gold nanoparticle aggregate films. Appl. Phy. Lett. 2015, 107, 141111.

11. Nyk, M.; Wawrzynczyk, D.; Szeremeta, J.; Samoc, M. Spectrally resolved size-dependent third-order nonlinear optical properties of colloidal CdSe quantum dots. Appl. Phys. Lett. 2012, 100, 041102.

12. Pan, H.; Chu, H.; Li, Y.; Qi, N.; Zhao, S.; Li, G.; Li, D. Nonlinear optical properties of colloidal CdSe/ZnS quantum dots in PMMA. Nanotechnology 2020, 31, 195703.

13. Song, H.; Zhai, Y.Y.; Zhou, Z.K.; Hao, Z.H.; Zhou, L. Optical Nonlinearity of CdSe and CdSe-C 60 Quantum Dot. Mod. Phys. Lett. B 2008, 22, 3207-3213.

14. Wang, X.; Du, Y.; Ding, S.; Wang, Q.; Xiong, G.; Xie, M.; Shen, X.; Pang, D. Preparation and Third-Order Optical Nonlinearity of Self-Assembled Chitosan/CdSe- ZnS Core- Shell Quantum Dots Multilayer Films. J. Phys. Chem. B 2006, 110, 1566-1570.

15. Dancus, I.; Popescu, S.T.; Petris, A. Single shot interferometric method for measuring the nonlinear refractive index. Opt. Express 2013, 21, 31303. doi:10.1364/OE.21.031303.

16. Zhu, L.; Sun, M.; Chen, J.; Yu, J.; Zhou, C. Integrated digital holography for measuring the photothermal effect induced by femtosecond laser pulses. Opt. Eng. 2014, 53, 112311. doi:10.1117/1.OE.53.11.112311.

17. Momgaudis, B.; Guizard, S.; Bilde, A.; Melninkaitis, A. Nonlinear refractive index measurements using time-resolved digital holography. Opt. Lett. 2018, 43, 304. doi:10.1364/OL.43.000304.

18. Momgaudis, B.; Kudriasov, V.; Vengris, M.; Melninkaitis, A. Quantitative assessment of nonlinearly absorbed energy in fused silica via time-resolved digital holography. Opt. Express 2019, 27, 7699. doi:10.1364/OE.27.007699.

19. Gomes, J.; Barbano, E.; Misoguti, L. Cross-section profile of the nonlinear refractive index of Gorilla Glass obtained by nonlinear ellipse rotation measurements. Appl. Opt. 2019, 58, 7858-7861.

20. Gomes, J.; Barbano, E.; Zilio, S.; Misoguti, L. Observation of strong local inhomogeneity of the optical nonlinearities on polycrystalline zincblende semiconductors. Nonlinear Optics. Optical Society of America, 2019, pp. NTu4A-32.

21. Petrov, N.; Nalegaev, S.; Belashov, A.; Shevkunov, I.; Putilin, S.; Lin, Y.; Cheng, C. Time-resolved inline digital holography for the study of noncollinear degenerate phase modulation. Opt. Lett. 2018, 43, 3481-3484.

22. Sheik-Bahae, M.; Wang, J.; DeSalvo, R.; Hagan, D.J.; Van Stryland, E.W. Measurement of nondegenerate nonlinearities using a two-color Z scan. Opt. Lett. 1992, 17, 258. doi:10.1364/OL.17.000258.

23. Momgaudis, B.; Guizard, S.; Bilde, A.; Melninkaitis, A. Nonlinear refractive index measurements using time-resolved digital holography. Opt. Lett. 2018, 43, 304-307. doi:10.1364/OL.43.000304.

24. Miguez, M.; Barbano, E.; Zilio, S.C.; Misoguti, L. Accurate measurement of nonlinear ellipse rotation using a phase-sensitive method. Opt. Express 2014, 22, 25530-25538.

25. Beygi, M.G.; Karimzadeh, R.; Dashtdar, M. Nonlinear refractive index measurement by Fresnel diffraction from phase object. Opt. Laser Technol. 2015, 66, 151-155. doi:10.1016/j.optlastec.2014.09.004.

26. Nabiev, I.; Sukhanova, A.; Even-Desrumeaux, K.; Chames, P.; Baty, D.; Artemyev, M.; Oleinikov, V. Engineering of ultra-small diagnostic nanoprobes through oriented conjugation of single-domain antibodies and quantum dots. Protoc. Exch. $2012,10$.

27. Petrov, N.V.; Putilin, S.E.; Chipegin, A.A. Time-resolved image plane off-axis digital holography. Appl. Phys. Lett. 2017, 110, 161107. doi:10.1063/1.4981899.

28. Lu, X.; Liu, Q.; Liu, Z.; Sun, S.; Ding, P.; Ding, B.; Hu, B. Measurement of nonlinear refractive index coefficient using emission spectrum of filament induced by gigawatt-femtosecond pulse in BK7 glass. Appl. Opt. 2012, 51, 2045-2050.

29. Belashov, A.V.; Cheng, C.J.; Petrov, N.V. Noncollinear degenerate phase modulation in samples with inhomogeneous optical nonlinear properties. Appl. Opt. 2021, 60, B14-B22.

30. Belashov, A.; Nalegaev, S.; Shevkunov, I.; Putilin, S.; Lin, Y.; Cheng, C.; Petrov, N. Experimental evaluation of inhomogeneous nonlinear refractive index distribution using time-resolved inline digital holography. Frontiers in Optics. Optical Society of America, 2021, pp. JW7A-119.

31. Mathew, S.; Saran, A.D.; Singh Bhardwaj, B.; Ani Joseph, S.; Radhakrishnan, P.; Nampoori, V.; Vallabhan, C.; Bellare, J.R. Size dependent optical properties of the CdSe-CdS core-shell quantum dots in the strong confinement regime. J. Appl. Phys. 2012, $111,074312$. 